University of Wollongong

Research Online

Faculty of Engineering and Information

Faculty of Engineering and Information

Sciences - Papers: Part A

Sciences

$1-1-2013$

Direct approach for solving nonlinear evolution and two-point boundary value problems

Jonu Lee

University of Wollongong

Rathinasamy Sakthivel

Sungkyunkwan University

Follow this and additional works at: https://ro.uow.edu.au/eispapers

Part of the Engineering Commons, and the Science and Technology Studies Commons

Research Online is the open access institutional repository for the University of Wollongong. For further information contact the UOW Library: research-pubs@uow.edu.au 


\title{
Direct approach for solving nonlinear evolution and two-point boundary value problems
}

\begin{abstract}
Time-delayed nonlinear evolution equations and boundary value problems have a wide range of applications in science and engineering. In this paper, we implement the differential transform method to solve the nonlinear delay differential equation and boundary value problems. Also, we present some numerical examples including time-delayed nonlinear Burgers equation to illustrate the validity and the great potential of the differential transform method. Numerical experiments demonstrate the use and computational efficiency of the method. This method can easily be applied to many nonlinear problems and is capable of reducing the size of computational work.
\end{abstract}

\section{Keywords}

value, boundary, point, two, solving, approach, problems, evolution, direct, nonlinear

\section{Disciplines}

Engineering | Science and Technology Studies

\section{Publication Details}

Lee, J. \& Sakthivel, R. (2013). Direct approach for solving nonlinear evolution and two-point boundary value problems. Pramana: journal of physics, 81 (6), 893-909. 


\begin{tabular}{|c|}
\hline $\begin{array}{r}\text { PRAMANA } \\
\text { - journal of } \\
\text { physics }\end{array}$ \\
\hline
\end{tabular}

\title{
Direct approach for solving nonlinear evolution and two-point boundary value problems
}

\author{
JONU LEE ${ }^{1}$ and RATHINASAMY SAKTHIVEL ${ }^{2, *}$ \\ ${ }^{1}$ School of Mathematics and Applied Statistics, University of Wollongong, Wollongong, \\ NSW 2522, Australia \\ ${ }^{2}$ Department of Mathematics, Sungkyunkwan University, Suwon 440-746, \\ South Korea \\ *Corresponding author. E-mail: krsakthivel@yahoo.com
}

MS received 26 December 2011; revised 11 January 2013; accepted 7 October 2013

DOI: 10.1007/s12043-013-0629-x; ePublication: 1 December 2013

\begin{abstract}
Time-delayed nonlinear evolution equations and boundary value problems have a wide range of applications in science and engineering. In this paper, we implement the differential transform method to solve the nonlinear delay differential equation and boundary value problems. Also, we present some numerical examples including time-delayed nonlinear Burgers equation to illustrate the validity and the great potential of the differential transform method. Numerical experiments demonstrate the use and computational efficiency of the method. This method can easily be applied to many nonlinear problems and is capable of reducing the size of computational work.
\end{abstract}

Keywords. Differential transform method; series solutions; time-delayed Burgers equation; boundary value problems.

PACS Nos 02.30.Jr; 02.30.Ik; 02.30Hq

\section{Introduction}

The nonlinear evolution equations and boundary value problems have become one of the central theme of perpetual interest in mathematical physics. In order to understand better the nonlinear phenomena as well as their further applications in the real-life situations, it is important to find their exact solutions. Accurate and fast numerical solution of nonlinear equations is of great importance due to their wide applications in scientific and engineering research. Different numerical methods have been proposed by various authors for solving nonlinear problems such as exp-function method [1-6], Jacobi elliptic function method [7], variational iteration method [8,9], tanh function method [10,11], $\left(G^{\prime} / G\right)$ expansion method [12], homotopy perturbation method [13-15] and so on. However, 


\section{Jonu Lee and Rathinasamy Sakthivel}

practically there is no unified method that can be used to handle all types of nonlinearities. Another important method used to obtain exact solutions of nonlinear problem is the differential transform method (DTM) $[16,17]$. The concept of the DTM was first proposed by Zhou [17] to solve both linear and nonlinear initial value problems arising in electrical circuit analysis. DTM provides an efficient explicit and numerical solution with high accuracy, minimal calculations and without linearization. The DTM has been successfully implemented to many kinds of nonlinear problems and its applications can be found in [18-25] and references therein.

A number of nonlinear phenomena in many real-world problems are described by the interaction between convection and diffusion [26,27]. The well-known partial differential equation (PDE) having such interplay of reaction and diffusion is the Burgers equation [28]. The diffusion processes, however, get significantly modified when the memory effects are taken into account. This implies that the correlation between the successive movements of the diffusing particles may be understood as a delay in the flux for a given concentration gradient. Thus, existence of time delay is an important feature in convection diffusion systems. So, it is important and necessary to study the nonlinear evolution equations with delay term. In this paper, we consider the well-known generalization of the Burgers-Fisher equation, namely generalized time-delayed Burgers-Fisher equation of the form $[29,30]$

$$
\tau u_{t t}+\left[1-\tau f_{u}\right] u_{t}=u_{x x}-p u^{s} u_{x}+f(u), \quad f(u)=q u\left(1-u^{s}\right),
$$

where $p, q, s$ are constants and $\tau$ is a time-delay constant. Equation (1) reduces to the classical Burgers equation when $q=\tau=0$ and $p=s=1$.

On the other hand, the two-point boundary value problem occurs in a wide variety of problems in engineering and science, including the modelling of chemical reactions, heat transfer, diffusion, and the solution of optimal control problems (see $[31,32]$ and references therein). The most common numerical method for solving these problems is to use shooting methods [33,34]. Although shooting method has many advantages such as a fast solver and a reduced size of the system, it also requires a huge amount of computational work for obtaining accurate approximations, especially for nonlinear problems.

In this paper, we obtain exact solutions of the two-point boundary value problems of the form

$$
y^{\prime \prime}=f\left(x, y, y^{\prime}\right), \quad a<x<b
$$

subject to the boundary conditions

$$
y(a)=\alpha, \quad y(b)=\beta,
$$

where $f$ is continuous on the set $D=\left\{\left(x, y, y^{\prime}\right) \mid a \leq x \leq b, y, y^{\prime} \in R\right\}, a, b, \alpha$ and $\beta$ are constants.

The basic motivation of this paper is to implement the DTM to obtain numerical solution for the time-delayed nonlinear evolution equations and boundary value problems. Further, we present some numerical examples including linear as well as nonlinear boundary value problems. It is shown that the method provides the solution in rapid convergent series. The DTM has been shown to effectively, easily and accurately solve a large class of linear, non-linear partial differential equations with approximate solutions which converge very rapidly to accurate solutions. 


\section{DTM Method}

The basic definitions and fundamental operations of the two-dimensional differential transform are defined in $[35,36]$ as follows:

If function $w(x, y)$ is analytic and differentiated continuously with respect to time $y$ in the domain of interest, then

$$
W(k, h)=\frac{1}{k ! h !}\left[\frac{\partial^{k+h} w(x, y)}{\partial x^{k} \partial y^{h}}\right]_{(x=0, y=0)},
$$

where $w(x, y)$ is the original function and $W(k, h)$ is the transformed function, which is also called the $T$-function. In this paper, the lower and upper case letters represent the original and transformed functions, respectively. The differential inverse transform of $W(k, h)$ is defined as

$$
w(x, y)=\sum_{k=0}^{\infty} \sum_{h=0}^{\infty} W(k, h) x^{k} y^{k} .
$$

Combining eqs (4) and (5), we obtain

$$
w(x, y)=\sum_{k=0}^{\infty} \sum_{h=0}^{\infty} \frac{1}{k ! h !}\left[\frac{\partial^{k+h} w(x, y)}{\partial x^{k} \partial y^{h}}\right]_{(x=0, y=0)} x^{k} y^{k} .
$$

The fundamental operations of the two-dimensional differential transform method are listed in table 1.

\section{Time-delayed evolution equations}

In this section, we obtain the exact solutions for the time-delayed Burgers and BurgersFisher equations using the DTM method.

\subsection{Time-delayed Burgers equation}

Example 3.1. Consider the time-delayed Burgers equation of the form [29]

$$
\tau u_{t t}+u_{t}+u u_{x}-u_{x x}=0
$$

Table 1. The operators for the two-dimensional differential transform method.

$$
\begin{aligned}
& w(x, y)=u(x, y) \pm v(x, y) \\
& w(x, y)=\alpha u(x, y) \\
& w(x, y)=\frac{\partial u(x, y)}{\partial x} \\
& w(x, y)=\frac{\partial u(x, y)}{\partial y} \\
& w(x, y)=u(x, y) v(x, y) \\
& w(x, y)=\frac{\partial^{r+s} u(x, y)}{\partial x^{r} \partial y^{s}}
\end{aligned}
$$

$$
\begin{aligned}
W(k, h)= & U(k, h) \pm V(k, h) \\
W(k, h)= & \alpha U(k, h), \quad \alpha \text { is a constant } \\
W(k, h)= & (k+1) U(k+1, h) \\
W(k, h)= & (h+1) U(k, h+1) \\
W(k, h)= & \sum_{r=0}^{k} \sum_{s=0}^{h} U(r, h-s) V(k-r, s) \\
W(k, h)= & (k+1)(k+2) \cdots(k+r)(h+1)(h+2) \\
& \cdots(h+s) U(k+r, h+s)
\end{aligned}
$$




\section{Jonu Lee and Rathinasamy Sakthivel}

with the initial conditions

$$
u(x, 0)=1+2(1-\tau) \tan x
$$

and

$$
u_{t}(x, 0)=-2(1-\tau) \sec ^{2} x
$$

Solution: Taking the two-dimensional transform of eq. (7) by using the related definitions in table 1 , we obtain

$$
\begin{aligned}
& \tau(h+1)(h+2) U(k, h+2)+(h+1) U(k, h+1) \\
& \quad+\sum_{r=0}^{k} \sum_{s=0}^{h}(k-r+1) U(r, h-s) U(k-r+1, s) \\
& \quad-(k+1)(k+2) U(k+2, h)=0 .
\end{aligned}
$$

The initial conditions (8) can be transformed at $x=0$ as

$$
\begin{aligned}
U(k, 0) & = \begin{cases}1, & k=0 \\
0, & k=2,4,6, \ldots,\end{cases} \\
U(k, 1) & = \begin{cases}-2(1-\tau), & k=0 \\
0, & k=1,3,5, \ldots\end{cases}
\end{aligned}
$$

and

$$
\begin{aligned}
& U(1,0)=2(1-\tau), \quad U(3,0)=\frac{2(1-\tau)}{3}, \\
& U(5,0)=\frac{4(1-\tau)}{15}, \quad U(7,0)=\frac{34(1-\tau)}{315}, \ldots, \\
& U(2,1)=-2(1-\tau), \quad U(4,1)=-\frac{4(1-\tau)}{3}, \\
& U(6,1)=-\frac{34(1-\tau)}{45}, \quad U(8,1)=-\frac{124(1-\tau)}{315}, \ldots .
\end{aligned}
$$

Substituting (10), (11) and (12) in (9) and after some manipulations, we obtain the closed form solution of (7) and (8) as

$$
\begin{aligned}
u(x, t) & =\sum_{k=0}^{\infty} \sum_{h=0}^{\infty} U(k, h) x^{k} t^{h} \\
& =1+2(1-\tau)\left\{(x-t)+\frac{1}{3}(x-t)^{3}+\frac{2}{15}(x-t)^{5}+\frac{17}{315}(x-t)^{7}+\cdots\right\} \\
& =1+2(1-\tau) \tan (x-t),
\end{aligned}
$$

which is the exact solution of (7) and (8).

Example 3.2. Next consider eq. (7) for which the initial profile is taken as

$$
u(x, 0)=1+\tanh \left\{\frac{x}{2(\tau-1)}\right\}
$$


and

$$
u_{t}(x, 0)=-\frac{1}{2(\tau-1)} \operatorname{sech}^{2}\left\{\frac{x}{2(\tau-1)}\right\} .
$$

Solution: Taking two-dimensional transform of eq. (7) again by using table 1 and by applying the initial condition, eq. (14) the initial transformation coefficients are determined by

$$
\begin{aligned}
& U(k, 0)= \begin{cases}1, & k=0 \\
0, & k=2,4,6, \ldots\end{cases} \\
& U(k, 1)= \begin{cases}-\frac{1}{2(\tau-1)}, & k=0 \\
0, & k=1,3,5, \cdots\end{cases}
\end{aligned}
$$

and

$$
\begin{aligned}
& U(1,0)=\frac{1}{2(\tau-1)}, \quad U(3,0)=-\frac{1}{24(\tau-1)^{3}}, \\
& U(5,0)=\frac{1}{240(\tau-1)^{5}}, \quad U(7,0)=-\frac{17}{40320(\tau-1)^{7}}, \ldots, \\
& U(2,1)=\frac{1}{8(\tau-1)^{3}}, \quad U(4,1)=-\frac{1}{48(\tau-1)^{5}}, \\
& U(6,1)=\frac{17}{5760(\tau-1)^{7}}, \quad U(8,1)=-\frac{31}{80640(\tau-1)^{9}}, \ldots
\end{aligned}
$$

Substituting (15)-(17) in (9), we obtain the following closed form solution:

$$
\begin{aligned}
u(x, t)= & \sum_{k=0}^{\infty} \sum_{h=0}^{\infty} U(k, h) x^{k} t^{h} \\
= & 1+\frac{x-t}{2(\tau-1)}-\frac{1}{3}\left\{\frac{x-t}{2(\tau-1)}\right\}^{3}+\frac{2}{15}\left\{\frac{x-t}{2(\tau-1)}\right\}^{5} \\
& -\frac{17}{315}\left\{\frac{x-t}{2(\tau-1)}\right\}^{7}+\cdots \\
= & 1+\tanh \left\{\frac{x-t}{2(\tau-1)}\right\},
\end{aligned}
$$

which is the exact solution of (7) and (14).

\subsection{Time-delayed Burgers-Fisher equation}

In this subsection, we obtain the solution of the time-delayed Burgers-Fisher equation. When $p=s=q=1$, eq. (1) reduces to the form

$$
\tau u_{t t}+(1-\tau) u_{t}+2 \tau u u_{t}-u_{x x}+u u_{x}-u+u^{2}=0
$$




\section{Jonu Lee and Rathinasamy Sakthivel}

where $\tau$ is a time-delay constant. This equation may be called the time-delayed BurgersFisher equation. This equation shows a prototypical model for describing the interaction between the reaction mechanism, convection effect and diffusion transport [37]. It is clear that when $\tau=0$, eq. (19) reduces to the classical Burgers-Fisher equation discussed in [37].

Example 3.3. Consider the time-delayed Burgers-Fisher equation

$$
\tau u_{t t}+(1-\tau) u_{t}+2 \tau u u_{t}-u_{x x}+u u_{x}-u+u^{2}=0
$$

with the initial conditions

$$
u(x, 0)=\frac{1}{2}+\frac{1}{2} \tanh \left(\frac{\tau+1}{\tau-4} x\right)
$$

and

$$
u_{t}(x, 0)=-\frac{5}{4(\tau-4)} \operatorname{sech}^{2}\left(\frac{\tau+1}{\tau-4} x\right)
$$

Solution: The transformed version of eq. (20) can be written in the following recurrence formula:

$$
\begin{aligned}
& \tau(h+1)(h+2) U(k, h+2)+(1-\tau)(h+1) U(k, h+1) \\
&+2 \tau \sum_{r=0}^{k} \sum_{s=0}^{h}(s+1) U(r, h-s) U(k-r, s+1) \\
&-(k+1)(k+2) U(k+2, h) \\
&+\sum_{r=0}^{k} \sum_{s=0}^{h}(k-r+1) U(r, h-s) U(k-r+1, s) \\
&-U(k, h)+\sum_{r=0}^{k} \sum_{s=0}^{h} U(r, h-s) U(k-r, s)=0 .
\end{aligned}
$$

Following the same procedure which is outlined for the previous problems, the transformation coefficients can easily be evaluated. The initial conditions (21) can be transformed at $x=0$ as

$$
\begin{aligned}
& U(k, 0)= \begin{cases}1 / 2, & k=0 \\
0, & k=2,4,6, \ldots\end{cases} \\
& U(k, 1)= \begin{cases}-(5 / 4(\tau-4)), & k=0 \\
0, & k=1,3,5, \ldots\end{cases}
\end{aligned}
$$

and

$$
\begin{aligned}
& U(1,0)=\frac{\tau+1}{2(\tau-4)}, \quad U(3,0)=-\frac{(\tau+1)^{3}}{6(\tau-4)^{3}}, \\
& U(5,0)=\frac{(\tau+1)^{5}}{15(\tau-4)^{5}}, \quad U(7,0)=-\frac{17(\tau+1)^{7}}{630(\tau-4)^{7}}, \ldots,
\end{aligned}
$$




$$
\begin{aligned}
& U(2,1)=\frac{5(\tau+1)^{2}}{4(\tau-4)^{3}}, \quad U(4,1)=-\frac{5(\tau+1)^{4}}{6(\tau-4)^{5}}, \\
& U(6,1)=\frac{17(\tau+1)^{6}}{36(\tau-4)^{7}}, \quad U(8,1)=-\frac{31(\tau+1)^{8}}{126(\tau-4)^{9}}, \ldots .
\end{aligned}
$$

Substituting (23), (24) and (25) in (22), we obtain the following closed form solution:

$$
\begin{aligned}
u(x, t)= & \sum_{k=0}^{\infty} \sum_{h=0}^{\infty} U(k, h) x^{k} t^{h} \\
= & \frac{1}{2}+\frac{1}{2}\left[\frac{\tau+1}{\tau-4}\left(x-\frac{5 t}{2(\tau+1)}\right)\right. \\
& -\frac{1}{3}\left\{\frac{\tau+1}{\tau-4}\left(x-\frac{5 t}{2(\tau+1)}\right)\right\}^{3}+\frac{2}{15}\left\{\frac{\tau+1}{\tau-4}\left(x-\frac{5 t}{2(\tau+1)}\right)\right\}^{5} \\
& \left.-\frac{17}{315}\left\{\frac{\tau+1}{\tau-4}\left(x-\frac{5 t}{2(\tau+1)}\right)\right\}^{7}+\cdots\right] \\
= & \frac{1}{2}+\frac{1}{2} \tanh \left\{\frac{\tau+1}{\tau-4}\left(x-\frac{5 t}{2(\tau+1)}\right)\right\}
\end{aligned}
$$

which is the exact solution of (20) and (21).

\section{Boundary value problems}

In this section, DTM is applied to solve two-point boundary value problems. To study the accuracy and efficiency of this method, it is applied to four problems involving linear and nonlinear terms, and the numerical results are presented.

Example 4.1. We consider the following linear boundary value problem [33,34]:

$$
k y^{\prime \prime}-x y^{\prime}-y=-\left(1+k \pi^{2}\right) \cos (\pi x)+\pi x \sin (\pi x), 0<x<1
$$

with boundary conditions

$$
y(0)=1, \quad y(1)=-1 .
$$

Solution: Applying the differential transform to eq. (27), we obtain

$$
\begin{aligned}
& k(h+1)(h+2) Y(h+2) \\
& \quad-\sum_{l=0}^{h} \delta(l-1)(h-l+1) Y(h-l+1)-Y(h)=-\left(1+k \pi^{2}\right) \frac{\pi^{h}}{h !} \cos \left(\frac{\pi h}{2}\right) \\
& \quad+\pi \sum_{l=0}^{h} \delta(l-1) \frac{\pi^{h-l}}{(h-l) !} \sin \left(\frac{\pi(h-l)}{2}\right) .
\end{aligned}
$$


The boundary conditions in eq. (28) can be transformed at $x=0$ as

$$
Y(0)=1, \quad Y(1)=a
$$

and

$$
\sum_{h=0}^{\infty} Y(h)=-1
$$

It follows from eqs (29) and (30) that

$$
\begin{aligned}
& Y(2)=-\frac{\pi^{2}}{2 !}, \quad Y(3)=\frac{a}{3 k}, \quad Y(4)=\frac{\pi^{4}}{4 !}, \quad Y(5)=\frac{a}{15 k}, \\
& Y(6)=-\frac{\pi^{6}}{6 !}, \quad Y(7)=\frac{a}{105 k^{3}}, \ldots .
\end{aligned}
$$

So we have the following:

$$
Y(h)= \begin{cases}(-1)^{h / 2}\left(\pi^{h} / h !\right), & h=0,2,4,6, \ldots \\ a / \prod_{n=0}^{(h-1) / 2}(2 n+1) k^{(h-1) / 2}, & h=1,3,5,7, \ldots .\end{cases}
$$

From the third condition of eq. (30), we can find the constant $a$ as follows:

$$
\sum_{h=2 i}^{\infty}(-1)^{h / 2} \frac{\pi^{h}}{h !}+\sum_{h=2 i+1}^{\infty} \frac{a}{\prod_{n=0}^{(h-1) / 2}(2 n+1) k^{(h-1) / 2}}=-1,
$$

where $i$ is a nonnegative integer, $i=0,1,2, \ldots$. Equation (33) gives us the value of constant $a$

$$
\cos \pi+a \sum_{h=2 i+1}^{\infty} \frac{1}{\prod_{n=0}^{(h-1) / 2}(2 n+1) k^{(h-1) / 2}}=-1,
$$

we have $a=0$. Now eq. (32) can be rewritten as

$$
Y(h)= \begin{cases}(-1)^{h / 2}\left(\pi^{h} / h !\right), & h=0,2,4,6, \cdots \\ 0, & h=1,3,5,7, \ldots .\end{cases}
$$

Finally, we obtain the following closed form solution:

$$
\begin{aligned}
y(x) & =\sum_{h=0}^{\infty} Y(h) x^{h} \\
& =1-\frac{(\pi x)^{2}}{2 !}+\frac{(\pi x)^{4}}{4 !}-\frac{(\pi x)^{6}}{6 !}+\frac{(\pi x)^{8}}{8 !}-\cdots \\
& =\cos (\pi x) .
\end{aligned}
$$

Example 4.2. Next, we consider the following linear differential equation $[33,34]$ :

$$
k y^{\prime \prime}=y-\left(1+k \pi^{2}\right) \cos (\pi x), \quad 0<x<1
$$

with the boundary conditions

$$
y(0)=1+\exp \left(-\frac{1}{\sqrt{k}}\right), \quad y(1)=-1+\exp \left(-\frac{2}{\sqrt{k}}\right) .
$$


Solution: The transformed version of eq. (37) can be written in the following recurrence formula:

$$
k(h+1)(h+2) Y(h+2)=Y(h)-\left(1+k \pi^{2}\right) \frac{\pi^{h}}{h !} \cos \left(\frac{\pi h}{2}\right) .
$$

The boundary conditions in eq. (38) can be transformed at $x=0$ as

$$
Y(0)=1+\exp \left(-\frac{1}{\sqrt{k}}\right), \quad Y(1)=a
$$

and

$$
\sum_{h=0}^{\infty} Y(h)=-1+\exp \left(-\frac{2}{\sqrt{k}}\right) .
$$

Moreover, it follows from eqs (39) and (40) that

$$
\begin{array}{ll}
Y(2)=\frac{\exp (-1 / \sqrt{k})}{2 ! k}-\frac{\pi^{2}}{2 !}, & Y(3)=\frac{a}{3 ! k}, \\
Y(4)=\frac{\exp (-1 / \sqrt{k})}{4 ! k^{2}}+\frac{\pi^{4}}{4 !}, & Y(5)=\frac{a}{5 ! k^{2}}, \\
Y(6)=\frac{\exp (-1 / \sqrt{k})}{6 ! k^{3}}-\frac{\pi^{6}}{6 !}, & Y(7)=\frac{a}{7 ! k^{3}}, \ldots
\end{array}
$$

Now, we obtain the following:

$$
Y(h)= \begin{cases}\frac{\exp (-1 / \sqrt{k})}{h ! k^{h / 2}}+(-1)^{h / 2}\left(\pi^{h} / h !\right), & h=0,2,4,6, \ldots . \\ \frac{a}{h ! k^{(h-1) / 2}}, & h=1,3,5,7, \ldots .\end{cases}
$$

From the third condition of eq. (40), we can find the constant $a$ as follows:

$$
\begin{aligned}
\sum_{h=2 i}^{\infty} & {\left[\frac{\exp (-1 / \sqrt{k})}{h ! k^{h / 2}}+(-1)^{h / 2} \frac{\pi^{h}}{h !}\right]+\sum_{h=2 i+1}^{\infty} \frac{a}{h ! k^{(h-1) / 2}} } \\
& =-1+\exp \left(-\frac{2}{\sqrt{k}}\right),
\end{aligned}
$$

where $i$ is a nonnegative integer, $i=0,1,2, \ldots$. Equation (43) gives us the value of constant $a$

$$
\begin{aligned}
& \exp \left(-\frac{1}{\sqrt{k}}\right) \cosh \left(\frac{1}{\sqrt{k}}\right)+\cos \pi+a \sqrt{k} \sinh \left(\frac{1}{\sqrt{k}}\right) \\
& =-1+\exp \left(-\frac{2}{\sqrt{k}}\right),
\end{aligned}
$$

using

$$
\cosh t=\frac{\mathrm{e}^{t}+\mathrm{e}^{-t}}{2} \quad \text { and } \quad \sinh t=\frac{\mathrm{e}^{t}-\mathrm{e}^{-t}}{2},
$$


we have $a=-\mathrm{e}^{-1 / \sqrt{k}} / \sqrt{k}$. Now eq. (42) can be written as

$$
Y(h)=\left\{\begin{array}{ll}
\frac{\exp (-1 / \sqrt{k})}{h ! k^{h / 2}}+(-1)^{h / 2} \frac{\pi^{h}}{h !}, & h=0,2,4,6, \ldots . \\
-\frac{\exp (-1 / \sqrt{k})}{h ! k^{h / 2}}, & h=1,3,5,7, \ldots .
\end{array} .\right.
$$

Finally, we obtain the following exact solution:

$$
\begin{aligned}
y(x) & =\sum_{h=0}^{\infty} Y(h) x^{h} \\
& =\exp \left(-\frac{1}{\sqrt{k}}\right) \sum_{h=0}^{\infty} \frac{(-1)^{h}}{h !}\left(\frac{x}{\sqrt{k}}\right)^{h}+\sum_{h=2 i}^{\infty}(-1)^{h / 2} \frac{(\pi x)^{h}}{h !} \\
& =\exp \left(-\frac{x+1}{\sqrt{k}}\right)+\cos (\pi x),
\end{aligned}
$$

where $i$ is a nonnegative integer.

Example 4.3. Consider the following linear differential equation $[33,34]$ :

$$
y^{\prime \prime}-4 y=4 \cosh (1), \quad 0<x<1
$$

with the boundary conditions

$$
y(0)=y(1)=0 .
$$

Solution: The transformed version of eq. (48) can be written as

$$
\begin{aligned}
& 2 Y(2)-4 Y(0)=4 \cosh (1), \\
& (h+1)(h+2) Y(h+2)-4 Y(h)=0 \quad \text { for } h=1,2,3, \ldots .
\end{aligned}
$$

The boundary conditions in eq. (49) can be transformed at $x=0$ as

$$
Y(0)=0, \quad Y(1)=a \quad \text { and } \quad \sum_{h=0}^{\infty} Y(h)=0 .
$$

Also, it follows from eqs (50) and (51) that

$$
\begin{aligned}
& Y(2)=\frac{4 \cosh (1)}{2 !}, \quad Y(3)=\frac{4 a}{3 !}, \quad Y(4)=\frac{4^{2} \cosh (1)}{4 !}, \\
& Y(5)=\frac{4^{2} a}{5 !}, \quad Y(6)=\frac{4^{3} \cosh (1)}{6 !}, \quad Y(7)=\frac{4^{3} a}{7 !}, \ldots
\end{aligned}
$$

So we get the following:

$$
Y(h)= \begin{cases}0, & h=0 \\ \frac{2^{h-1} a}{h !}, & h=1,3,5,7, \ldots . \\ \frac{2^{h} \cosh (1)}{h !}, & h=2,4,6,8, \ldots .\end{cases}
$$


From the third condition of eq. (51), we can find the constant $a$. Now, we have

$$
a \sum_{h=2 i-1}^{\infty} \frac{2^{h-1}}{h !}+\cosh (1) \sum_{h=2 i}^{\infty} \frac{2^{h}}{h !}=0
$$

where $i$ is a positive integer, $i=1,2,3, \ldots$. Equation (54) gives us the value of constant $a$

$$
\begin{aligned}
& \frac{a}{2} \sum_{h=2 i-1}^{\infty} \frac{2^{h}}{h !}+\cosh (1) \sum_{h=2 i}^{\infty} \frac{2^{h}}{h !}=0, \\
& \frac{a \sinh (2)}{2}+\cosh (1)(-1+\cosh (2))=0,
\end{aligned}
$$

we have $a=-2 \sinh (1)$. Then eq. (53) can be written as

$$
Y(h)=\left\{\begin{array}{cl}
0, & h=0 \\
-\frac{2^{h} \sinh (1)}{h !}, & h=1,3,5,7, \ldots . \\
\frac{2^{h} \cosh (1)}{h !}, & h=2,4,6,8, \ldots .
\end{array}\right.
$$

Finally, we obtain the following closed form solution:

$$
\begin{aligned}
y(x) & =\sum_{h=0}^{\infty} Y(h) x^{h} \\
& =-\sinh (1) \sum_{h=2 i-1}^{\infty} \frac{(2 x)^{h}}{h !}+\cosh (1) \sum_{h=2 i}^{\infty} \frac{(2 x)^{h}}{h !} \\
& =-\sinh (1) \sinh (2 x)+\cosh (1) \cosh (2 x)-\cosh (1) \\
& =\cosh (2 x-1)-\cosh (1),
\end{aligned}
$$

where $i$ is a positive integer.

Example 4.4. Finally, we consider the following nonlinear boundary value problem [38]:

$$
u^{\prime \prime}(x)-u^{2}(x)=2 \pi^{2} \cos (2 \pi x)-\sin ^{4}(\pi x), \quad 0<x<1
$$

with the boundary conditions

$$
u(0)=u(1)=0 \text {. }
$$

Solution: The transformed version of eq. (59) can be written as

$$
\begin{aligned}
U(k+2)= & \frac{1}{(k+1)(k+2)} \sum_{r=0}^{k} U(r) U(k-r) \\
& +\frac{2^{k+1} \pi^{k+2}}{(k+2) !} \cos \left(\frac{k \pi}{2}\right)-\frac{V(k)}{(k+1)(k+2)},
\end{aligned}
$$


where $V(k)=0, k=1,3,5, \ldots$ and

$$
\begin{aligned}
& V(0)=0, \quad V(2)=0, \quad V(4)=\pi^{4}, \quad V(6)=-\frac{2 \pi^{6}}{3}, \\
& V(8)=\frac{\pi^{8}}{5}, \quad V(10)=-\frac{34 \pi^{10}}{945}, \ldots .
\end{aligned}
$$

The boundary conditions (60) can be transformed at $x=0$ as

$$
U(0)=0, \quad U(1)=a,
$$

where $a$ is an unknown constant to be determined later.

Substituting (62) and (63) in (61), we can obtain the following term

$$
\begin{aligned}
u(x)= & \sum_{k=0}^{\infty} U(k) x^{k} \\
= & a x+\pi^{2} x^{2}+\left(\frac{a^{2}}{12}-\frac{\pi^{4}}{3}\right) x^{4}+\frac{a \pi^{2}}{10} x^{5} \\
& +\frac{2 \pi^{6}}{45} x^{6}+\left(\frac{a^{3}}{252}-\frac{a \pi^{4}}{63}\right) x^{7}+\left(\frac{11 a^{2} \pi^{2}}{1680}-\frac{\pi^{8}}{315}\right) x^{8} \\
& +\left(\frac{a \pi^{4}}{360}+\frac{a \pi^{6}}{810}\right) x^{9}+\left(\frac{a^{4}}{6048}-\frac{11 a^{2} \pi^{4}}{11340}+\frac{2 \pi^{10}}{14175}\right) x^{10}+\cdots .
\end{aligned}
$$

Now we obtain the following series solution up to $O\left(x^{11}\right)$ :

$$
\begin{aligned}
& \hat{u}_{1}(x)=\pi x+O\left(x^{2}\right) \\
& u_{1}^{2}(x)=\pi^{2} x^{2}+O\left(x^{3}\right) \\
& \hat{u}_{2}(x)=\pi x-\frac{\pi^{3}}{3 !} x^{3}+O\left(x^{4}\right), \\
& u_{2}^{2}(x)=\pi^{2} x^{2}-\frac{\pi^{4}}{3} x^{4}+O\left(x^{5}\right), \\
& \hat{u}_{3}(x)=\pi x-\frac{\pi^{3}}{3 !} x^{3}+\frac{\pi^{5}}{5 !} x^{5}+O\left(x^{6}\right), \\
& u_{3}^{2}(x)=\pi^{2} x^{2}-\frac{\pi^{4}}{3} x^{3}+\frac{2 \pi^{6}}{45} x^{6}+O\left(x^{7}\right), \\
& \hat{u}_{4}(x)=\pi x-\frac{\pi^{3}}{3 !} x^{3}+\frac{\pi^{5}}{5 !} x^{5}-\frac{\pi^{7}}{7 !} x^{7}+O\left(x^{8}\right), \\
& u_{4}^{2}(x)=\pi^{2} x^{2}-\frac{\pi^{4}}{3} x^{3}+\frac{2 \pi^{6}}{45} x^{6}-\frac{\pi^{8}}{315} x^{8}+O\left(x^{9}\right), \\
& \hat{u}_{5}(x)=\pi x-\frac{\pi^{3}}{3 !} x^{3}+\frac{\pi^{5}}{5 !} x^{5}-\frac{\pi^{7}}{7 !} x^{7}+\frac{\pi^{9}}{9 !} x^{7}+O\left(x^{10}\right), \\
& u_{5}^{2}(x)=\pi^{2} x^{2}-\frac{\pi^{4}}{3} x^{3}+\frac{2 \pi^{6}}{45} x^{6}-\frac{\pi^{8}}{315} x^{8}+\frac{2 \pi^{10}}{14175}+O\left(x^{11}\right),
\end{aligned}
$$


Table 2. The DTM results for $y(x)$ for the first four term approximations in comparison with the analytical solutions when $k=2$ for Example 4.2.

\begin{tabular}{lcccc}
\hline$x$ & $\left|y_{1}-y_{\text {DTM }}\right|$ & $\left|y_{2}-y_{\text {DTM }}\right|$ & $\left|y_{3}-y_{\text {DTM }}\right|$ & $\left|y_{4}-y_{\text {DTM }}\right|$ \\
\hline 0.1 & $2.3507275429 \mathrm{e}-09$ & $2.3507351264 \mathrm{e}-09$ & $2.9995954064 \mathrm{e}-06$ & $1.3173696289 \mathrm{e}-09$ \\
0.2 & $5.9981167446 \mathrm{e}-07$ & $5.9981360077 \mathrm{e}-07$ & $9.1863178944 \mathrm{e}-05$ & $1.3369774284 \mathrm{e}-06$ \\
0.3 & $1.5288676013 \mathrm{e}-05$ & $1.5288725002 \mathrm{e}-05$ & $6.6745351408 \mathrm{e}-04$ & $7.5962469603 \mathrm{e}-05$ \\
0.4 & $1.5155232705 \mathrm{e}-04$ & $1.5155281264 \mathrm{e}-04$ & $2.6901350652 \mathrm{e}-03$ & $1.3214389488 \mathrm{e}-03$ \\
0.5 & $8.9452299847 \mathrm{e}-04$ & $8.9452587078 \mathrm{e}-04$ & $7.8479194224 \mathrm{e}-03$ & $1.1988893688 \mathrm{e}-02$ \\
0.6 & $3.8007259812 \mathrm{e}-03$ & $3.8007382382 \mathrm{e}-03$ & $1.8654726969 \mathrm{e}-02$ & $7.1926331479 \mathrm{e}-02$ \\
0.7 & $1.2863373914 \mathrm{e}-02$ & $1.2863415667 \mathrm{e}-02$ & $3.8482708381 \mathrm{e}-02$ & $3.2391389343 \mathrm{e}-01$ \\
0.8 & $3.6839050271 \mathrm{e}-02$ & $3.6839170875 \mathrm{e}-02$ & $7.1529923126 \mathrm{e}-02$ & $1.1812234427 \mathrm{e}-00$ \\
0.9 & $9.2825621146 \mathrm{e}-02$ & $9.2825928290 \mathrm{e}-02$ & $1.2272207780 \mathrm{e}-01$ & $3.6635232949 \mathrm{e}-00$ \\
1.0 & $2.1135284298 \mathrm{e}-01$ & $2.1135355123 \mathrm{e}-01$ & $1.9754438069 \mathrm{e}-01$ & $9.9940046928 \mathrm{e}-00$ \\
\hline
\end{tabular}

Table 3. The DTM results for $y(x)$ for the first six term approximations in comparison with the analytical solutions when $k=2$ for Example 4.2.

\begin{tabular}{lcccc}
\hline$x$ & $\left|y_{1}-y_{\text {DTM }}\right|$ & $\left|y_{2}-y_{\text {DTM }}\right|$ & $\left|y_{3}-y_{\text {DTM }}\right|$ & $\left|y_{4}-y_{\text {DTM }}\right|$ \\
\hline 0.1 & $1.9285283585 \mathrm{e}-15$ & $1.9285283745 \mathrm{e}-15$ & $2.8882797569 \mathrm{e}-09$ & $8.5578714296 \mathrm{e}-15$ \\
0.2 & $7.8864206284 \mathrm{e}-12$ & $7.8864206936 \mathrm{e}-12$ & $3.5775803116 \mathrm{e}-07$ & $1.3952355892 \mathrm{e}-10$ \\
0.3 & $1.0204685621 \mathrm{e}-09$ & $1.0204685705 \mathrm{e}-09$ & $5.9147657323 \mathrm{e}-06$ & $4.0399398848 \mathrm{e}-08$ \\
0.4 & $3.2093837859 \mathrm{e}-08$ & $3.2093838123 \mathrm{e}-08$ & $4.2870852933 \mathrm{e}-05$ & $2.2416855468 \mathrm{e}-06$ \\
0.5 & $4.6476600840 \mathrm{e}-07$ & $4.6476601223 \mathrm{e}-07$ & $1.9774369039 \mathrm{e}-04$ & $5.0235147891 \mathrm{e}-05$ \\
0.6 & $4.1194644750 \mathrm{e}-06$ & $4.1194645089 \mathrm{e}-06$ & $6.8521902651 \mathrm{e}-04$ & $6.3373597706 \mathrm{e}-04$ \\
0.7 & $2.6012189622 \mathrm{e}-05$ & $2.6012189837 \mathrm{e}-05$ & $1.9488150259 \mathrm{e}-03$ & $5.3732785844 \mathrm{e}-03$ \\
0.8 & $1.2811596577 \mathrm{e}-04$ & $1.2811596683 \mathrm{e}-04$ & $4.7956607732 \mathrm{e}-03$ & $3.4038026631 \mathrm{e}-02$ \\
0.9 & $5.2180790241 \mathrm{e}-04$ & $5.2180790674 \mathrm{e}-04$ & $1.0564091767 \mathrm{e}-02$ & $1.7248635540 \mathrm{e}-01$ \\
1.0 & $1.8291040136 \mathrm{e}-03$ & $1.8291040289 \mathrm{e}-03$ & $2.1320118818 \mathrm{e}-02$ & $7.3264448681 \mathrm{e}-01$ \\
\hline
\end{tabular}

Table 4. The DTM results for $y(x)$ for the first ten term approximations in comparison with the analytical solutions when $k=2$ for Example 4.2.

\begin{tabular}{ccccc}
\hline$x$ & $\left|y_{1}-y_{\text {DTM }}\right|$ & $\left|y_{2}-y_{\text {DTM }}\right|$ & $\left|y_{3}-y_{\text {DTM }}\right|$ & $\left|y_{4}-y_{\text {DTM }}\right|$ \\
\hline 0.1 & $3.6039608644 \mathrm{e}-29$ & $3.6039608644 \mathrm{e}-29$ & $5.8991382824 \mathrm{e}-16$ & $1.6138010393 \mathrm{e}-26$ \\
0.2 & $3.7766065986 \mathrm{e}-23$ & $3.7766065986 \mathrm{e}-23$ & $1.1820292120 \mathrm{e}-12$ & $6.7542829561 \mathrm{e}-20$ \\
0.3 & $1.2544792313 \mathrm{e}-19$ & $1.2544792313 \mathrm{e}-19$ & $1.0003267365 \mathrm{e}-10$ & $5.0354578932 \mathrm{e}-16$ \\
0.4 & $3.9499348609 \mathrm{e}-17$ & $3.9499348609 \mathrm{e}-17$ & $2.3172356180 \mathrm{e}-09$ & $2.8088624801 \mathrm{e}-13$ \\
0.5 & $3.4194610196 \mathrm{e}-15$ & $3.4194610196 \mathrm{e}-15$ & $2.6391669541 \mathrm{e}-08$ & $3.7825421517 \mathrm{e}-11$ \\
0.6 & $1.3078757771 \mathrm{e}-13$ & $1.3078757771 \mathrm{e}-13$ & $1.9183282756 \mathrm{e}-07$ & $2.0720780242 \mathrm{e}-09$ \\
0.7 & $2.8464498417 \mathrm{e}-12$ & $2.8464498417 \mathrm{e}-12$ & $1.0227438732 \mathrm{e}-06$ & $6.0993309419 \mathrm{e}-08$ \\
0.8 & $4.0997944051 \mathrm{e}-11$ & $4.0997944051 \mathrm{e}-11$ & $4.3457534993 \mathrm{e}-06$ & $1.1391347820 \mathrm{e}-06$ \\
0.9 & $4.3077616973 \mathrm{e}-10$ & $4.3077616973 \mathrm{e}-10$ & $1.5526629443 \mathrm{e}-05$ & $1.5025785118 \mathrm{e}-05$ \\
1.0 & $3.5290799544 \mathrm{e}-09$ & $3.5290799544 \mathrm{e}-09$ & $4.8381681003 \mathrm{e}-05$ & $1.5061202092 \mathrm{e}-04$ \\
\hline
\end{tabular}




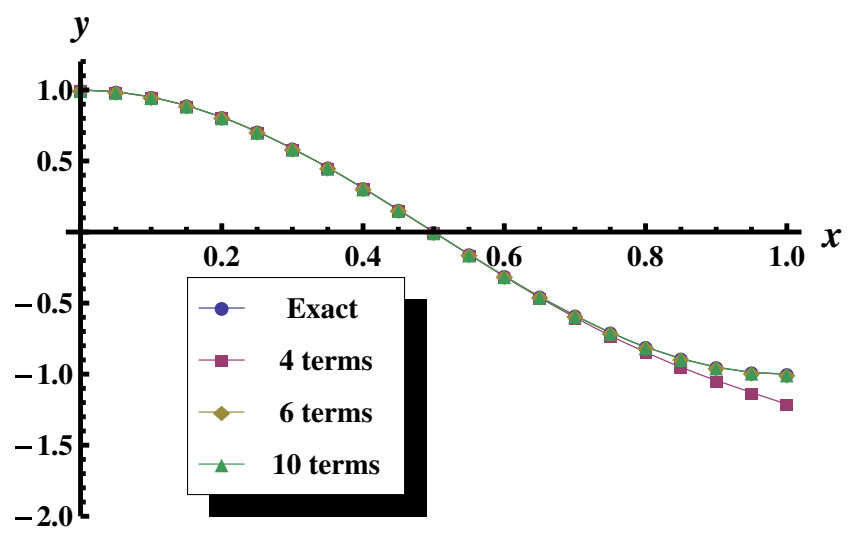

Figure 1. Exact solution and 4, 6, 10 terms approximation of Example 4.1.

From the above, we have

$$
\hat{u}(x)=\sum_{k=0}^{\infty} \frac{(\pi x)^{2 k+1}}{(2 k+1) !}=\sin (\pi x) .
$$

Now from the above solutions, we obtain

$$
\begin{aligned}
u(x)= & \sum_{k=0}^{\infty} U(k) x^{k} \\
= & a x+\frac{a^{2}}{12} x^{4}+\frac{a \pi^{2}}{10} x^{5}+\frac{a^{3}}{252} x^{7} \\
& +\frac{11 a^{2} \pi^{2}}{1680} x^{8}+\left(\frac{a \pi^{4}}{360}+\frac{a \pi^{6}}{810}\right) x^{9}+\cdots+\sin ^{2}(\pi x) .
\end{aligned}
$$

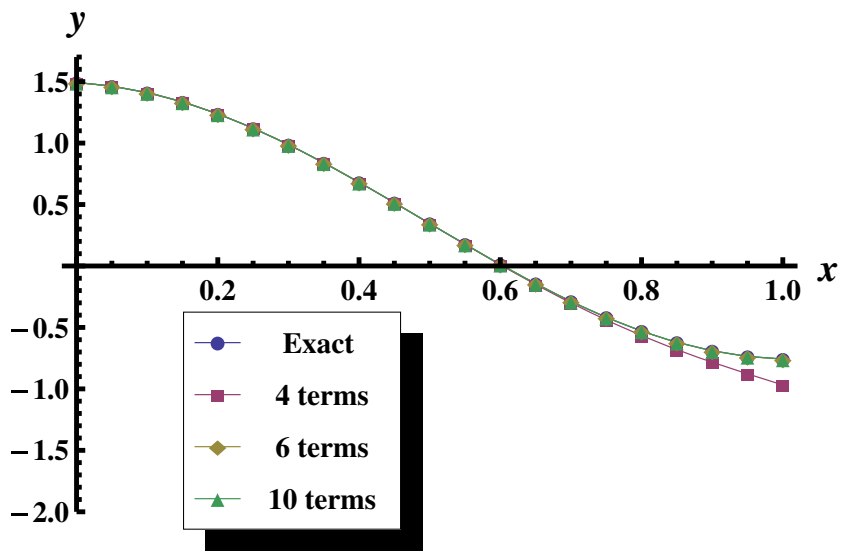

Figure 2. Exact solution and 4, 6, 10 terms approximation of Example 4.2. 


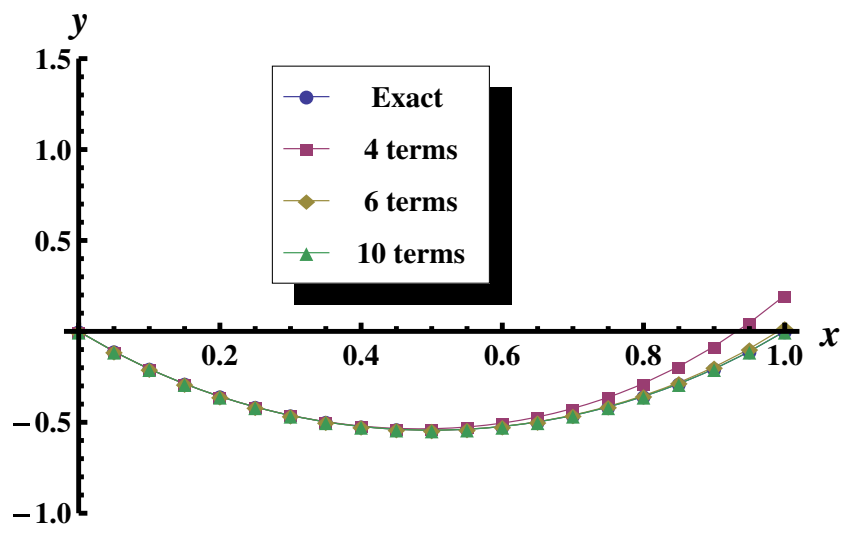

Figure 3. Exact solution and 4, 6, 10 terms approximation of Example 4.3.

The above solution must satisfy the boundary conditions. Imposing the boundary condition (60) at $x=1$ in the above equation, we obtain the value of the parameter $a=0$. Finally, we obtain the closed form solution as

$$
u(x)=\sin ^{2}(\pi x)
$$

which is the exact solution of eq. (59).

Further, to demonstrate the convergence of differential transform method, the numerical results of Example 4.2 are provided in tables 2, 3 and 4. It is concluded from tables 2, 3 and 4 that, the accuracy of the series solution is increased by computing more terms for

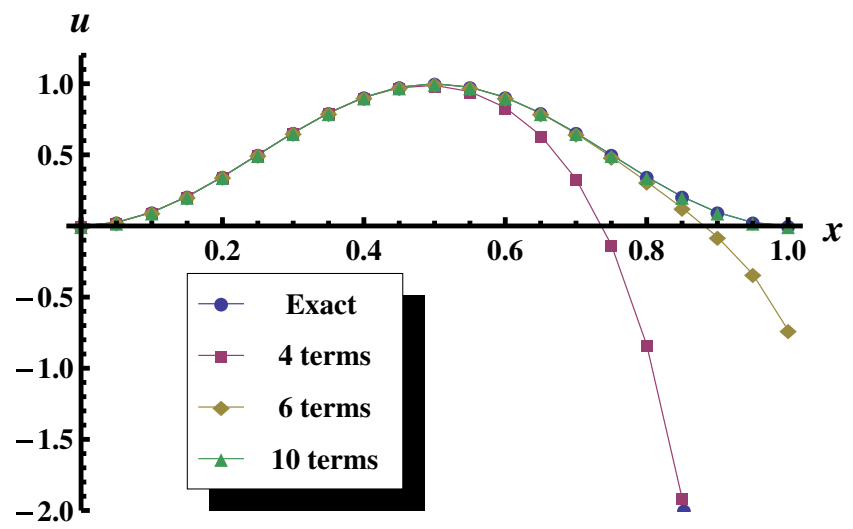

Figure 4. Exact solution and 4, 6, 10 terms approximation of Example 4.4. 


\section{Jonu Lee and Rathinasamy Sakthivel}

large values of $x$. Also, exact and approximate solutions of Examples 4.1, 4.2, 4.3 and 4.4 with 4, 6 and 10 term approximations are presented in figures 1-4.

\section{Conclusion}

In this article, the application of differential transform method was extended to obtain explicit solutions of the delay differential equations as well as boundary value problems. The method provides the solution as infinite series of functions with easily computable components. Several numerical experiments were considered to show the effectiveness of the method. As an application, time-delayed nonlinear Burgers equation was considered to illustrate the validity and the great potential of the differential transform method in solving delay differential equations. It is concluded that the method is very powerful and efficient in finding analytical solutions for a wide class of nonlinear equations.

\section{References}

[1] J H He and X H Wu, Chaos, Solitons and Fractals 30, 700 (2006)

[2] R Sakthivel and C Chun, Rep. Math. Phys. 62, 389 (2008)

[3] R Sakthivel and C Chun, Z. Naturforsch. A (Journal of Physical Sciences) 65a, 197 (2010)

[4] A Yildirim and Z Pinar, Comput. Math. Appl. 60, 1873 (2010)

[5] H Hosseini, M M Kabir and A Khajeh, Int. J. Nonlin. Sci. Numer. Simulat. 10, 1307 (2009)

[6] S Zhang and H Zhang, Comput. Math. Appl. 61, 1923 (2011)

[7] J Lee and R Sakthivel, Mod. Phys. Lett. B 24, 1011 (2010)

[8] J H He, Int. J. Non-Linear Mech. 34, 699 (1999)

[9] J H He and X H Wu, Comput. Math. Appl. 54, 881 (2007)

[10] J Lee and R Sakthivel, Commun. Math. Sci. 7, 1053 (2009)

[11] L Wazzan, Commun. Nonlinear Sci. Numer. Simulat. 14, 443 (2009)

[12] E M E Zayed and K A Gepreel, Int. J. Nonlin. Sci. Numer. Simulat. 11, 273 (2010)

[13] J H He, Comput. Methods Appl. Mech. Eng. 178, 257 (1999)

[14] A Saadatmandi, M Dehghan and A Eftekhari, Nonlinear Anal: Real World Applications 10, $1912(2009)$

[15] A Yildirim and D Agirseven, Int. J. Nonlin. Sci. Numer. Simulat. 10, 235 (2009)

[16] G E Pukhov, Differential transformations and mathematical modelling of physical processes (Naukova, Dumka, Kiev, 1986)

[17] J K Zhou, Differential transform and its applications for electrical circuits (Huazhong University Press, Wuhan, China, 1986)

[18] J Biazar and M Eslami, Phys. Lett. A 374, 2904 (2010)

[19] C K Chen and S H Ho, Appl. Math. Comput. 106, 171 (1999)

[20] S H Chang and I L Chang, Appl. Math. Comput. 195, 799 (2008)

[21] S H Chang and I L Chang, Appl. Math. Comput. 215, 2486 (2009)

[22] F Kangalgil and F Ayaz, Chaos, Solitons and Fractals 41, 464 (2009)

[23] M Kurulay and M Bayram, Commun. Nonlinear Sci. Numer. Simulat. 15, 1777 (2010)

[24] A S V Ravi Kanth and K Aruna, Phys. Lett. A 372, 6896 (2008)

[25] A S V Ravi Kanth and K Aruna, Comput. Phys. Commun. 180, 708 (2009)

[26] N F Briton, Reaction-diffusion equations and their applications to biology (Academic Press, 1986)

[27] J D Murray, Mathematical biology (Springer-Verlag, Berlin, 1993)

[28] J M Burgers, Adv. Appl. Mech. 1, 171 (1948) 
[29] E S Fahmy, H A Abdusalam and K R Raslan, Nonlin. Anal. 69, 4775 (2008)

[30] E S Fahmy, Chaos, Solitons and Fractals 38, 1209 (2008)

[31] M Dehghan and A Saadatmandi, Math. Comput. Model. 46, 1434 (2007)

[32] M Tatari and M Dehghan, Phys. Scr. 73, 672 (2006)

[33] K Keller, Numerical solutions of two-point boundary value problems (SIAM, Philadelphia, 1976)

[34] S M Roberts and J S Shipman, Two point boundary value problems: Shooting methods (American Elsevier, New York, 1972)

[35] F Ayaz, Appl. Math. Comput. 143, 361 (2003)

[36] F Ayaz, Appl. Math. Comput. 147, 547 (2004)

[37] W Xinyi and L Yuekai, Chin. Phys. Lett. 7, 145 (1990)

[38] L F Mo and S Q Wang, Nonlinear Anal. 71, e834 (2009) 